\title{
An interaction proteomics survey of transcription factor binding at recurrent TERT promoter mutations
}

\author{
Matthew M. Makowski ${ }^{1 *}$, Esther Willems ${ }^{1 *}$, Jun Fang ${ }^{3 * *}$, Jiyeon Choi ${ }^{3}$, Tongwu Zhang ${ }^{3}$, \\ Pascal W. T. C. Jansen ${ }^{1}$, Kevin M. Brown ${ }^{3 * *}$ and Michiel Vermeulen ${ }^{1,2}$ \\ ${ }^{1}$ Radboud Institute of Molecular Life Sciences, Radboud University Nijmegen, Nijmegen, The Netherlands \\ ${ }^{2}$ Cancer GenomiCs Netherlands, Utrecht, The Netherlands \\ ${ }^{3}$ Laboratory of Translational Genomics, Division of Cancer Epidemiology and Genetics, National Cancer Institute, \\ National Institutes of Health, Bethesda, MD, USA
}

\begin{abstract}
Aberrant telomerase reactivation in differentiated cells represents a major event in oncogenic transformation. Recurrent somatic mutations in the human telomerase reverse transcriptase (TERT) promoter region, predominantly localized to two nucleotide positions, are highly prevalent in many cancer types. Both mutations create novel consensus E26 transformation-specific (ETS) motifs and are associated with increased TERT expression. Here, we perform an unbiased proteome-wide survey of transcription factor binding at TERT promoter mutations in melanoma. We observe ELF1 binding at both mutations in vitro and we show that increased recruitment of GABP is enabled by the spatial architecture of native and novel ETS motifs in the TERT promoter region. We characterize the dynamics of competitive binding between ELF1 and GABP and provide evidence for ELF1 exclusion by transcriptionally active GABP. This study thus provides an important description of proteome-wide, mutation-specific binding at the recurrent, oncogenic TERT promoter mutations.
\end{abstract}

\section{Keywords:}

Biomedicine / Melanoma / Telomerase / Transcription

Additional supporting information may be found in the online version of this article at the publisher's web-site

\section{Introduction}

Telomerase reactivation, associated with senescence bypass and essentially unlimited proliferative capacity, is a classic hallmark of cancer [1,2]. However, because telomerase is typically silenced in differentiated cells, telomerase reactivation is thought to proceed through transcriptional mechanisms [3]. A number of factors have previously been implicated in TERT reactivation via various transcriptional pathways [4-6]. Importantly, two landmark studies identified two recurrent

Correspondence: Dr. M. Vermeulen, Radboud Institute of Molecular Life Sciences, Radboud University Nijmegen, Geert Grooteplein 28, 6525 GA Nijmegen, The Netherlands

E-mail: michiel.vermeulen@science.ru.nl

Abbreviations: ELF1, E74-like factor 1; ELF2, E74-like factor 2; ETS, E26 transformation-specific; ETV6, ETS Variant 6; GABP, GAbinding protein; TERT, Telomerase reverse transcriptase
Received: August 11, 2015

Revised: October 21, 2015

Accepted: November 5, 2015 somatic mutations (C228T and C250T) in the TERT promoter region in melanoma and other cancers $[7,8]$. Subsequent work confirmed TERT promoter mutations occur with high frequency in many cancer subtypes, in some cases approaching or exceeding the penetrance of canonical driver coding mutations $[9,10]$. Genome-wide analysis further confirmed TERT promoter mutations as the most consistently significant mutational hotspot across various cancer subtypes $[11,12]$. Intriguingly, the two TERT promoter mutations appear to be mutually exclusive, indicating potential functional redundancy $[7,8]$.

More recent work has begun to unravel the functional mechanisms by which TERT promoter mutations exert their oncogenic effect. For example, TERT promoter mutations were recently implicated in overcoming

\footnotetext{
*These authors contributed equally to this work.

**Additional corresponding author: Kevin M. Brown,

E-mail: Kevin.Brown3@nih.gov

Colour Online: See the article online to view Fig. 4 in colour.
} 


\section{Significance of the study}

Somatic mutations in the TERT promoter associated with TERT reactivation during oncogenesis are common and highly frequent in many different cancer subtypes. Understanding the mechanisms by which TERT promoter mutations exert their transcriptional effect is crucial towards clarifying their role in oncogenesis. Therefore, uncovering the ensemble of transcription factors that bind specifically to recurrent TERT promoter mutations directly implicates potential upstream effectors in TERT reactivation.
Furthermore, transcription factors that bind specifically at similar motifs can actively compete for binding at the same site in vivo. Therefore, characterizing the biochemical properties of competitive transcription factor binding at TERT promoter mutations reveals a complex and dynamic pattern of regulation at these sites. Ultimately, this study furthers our understanding of specific transcription factor binding at TERT promoter mutations and contributes to our knowledge of transcriptional regulation in oncogenic TERT reactivation. differentiation-associated transcriptional silencing of TERT expression, effectively extending telomere length and potentially contributing to tumorigenic immortalization $[13,14]$. Additionally, both mutations create novel E26 transformation-specific (ETS) transcription factor motifs within the TERT promoter region $[7,8]$. However, the exact factor or factors influencing TERT expression specifically via promoter mutation sites remained elusive until a recent study demonstrated that GABP, an ETS-family transcription factor, was a major functional interactor [15]. The identification of GABP as a mutation-specific interactor is particularly interesting, as multiple transcriptionally active binding modes for GABP have been previously been reported, including a heterodimer (GABPA/B) and a heterotetramer $\left(\mathrm{GABPA}_{2} / \mathrm{B}_{2}\right)$ [16-20]. The heterotetrameric binding mode of GABP was associated with TERT promoter mutation specific interactions. In particular, a precise spatial architecture between native ETS motifs and novel, mutation-specific ETS motifs was indicated as crucial for tetrameric GABP binding [15]. Very recently, another report indicated that mutation specific GABP binding is concurrent with a switch to active chromatin marks [21].

Despite progress in elucidating the molecular function of TERT promoter mutations, most of the studies thus far have targeted ETS-family factors. Complementary to targeted molecular studies, our group and others have previously established workflows for AP-MS/MS based identification of sequence specific protein-DNA binding on a proteome-wide scale $[22,23]$. Here, we perform an unbiased, proteome-wide survey of TERT promoter mutation specific transcription factor binding. We identify specific binding of multiple factors, including ELF1/2 and ETV6, and we confirm GABP as a direct, specific interactor in melanoma. We provide further characterization of the spatial architecture that promotes GABP binding at native and novel ETS motifs in the TERT promoter. Additionally, we analyze competitive binding dynamics between ELF1 and GABP and propose a model in which multimeric GABP binding to a native and novel ETS motif excludes ELF1 and activates TERT expression. In sum, this study provides an important molecular characterization of proteome-wide transcription factor binding events that are specific for recurrent, somatic mutations in the TERT promoter, further extending our knowledge of the oncogenic properties of this locus.

\section{Materials and methods}

\subsection{Cell culture and nuclear lysate extraction}

Melanoma cell lines were grown in adherent culture in RPMI 1640 (Gibco) supplemented with 10\% FBS, 200 mM HEPES (pH 7.9), $100 \mathrm{U} / \mathrm{mL}$ penicilin and $100 \mu \mathrm{g} / \mathrm{mL}$ streptomycin (Gibco).

Nuclear lysates were collected essentially as described previously [23]. Briefly, cells were incubated in hypotonic Buffer A (10 mM HEPES (pH 7.9), $1.5 \mathrm{mM} \mathrm{MgCl2,} 10 \mathrm{mM} \mathrm{KCl}$ and $0.15 \%$ NP40). Cells were then lysed by dounce homogenizer. Crude nuclei were collected by centrifugation and lysed in Buffer C (420 mM NaCl2, 20 mM HEPES (pH 7.9), 20\% (v/v) glycerol, $2 \mathrm{mM} \mathrm{MgCl}$, $0.2 \mathrm{mM}$ EDTA, 0.1\% NP40, EDTAfree complete protease inhibitors (Roche), and $0.5 \mathrm{mM}$ DTT) by rotation for $1 \mathrm{~h}$ at $4^{\circ} \mathrm{C}$. Nuclear lysates were collected as the soluble fraction, snap frozen in liquid nitrogen, and stored at $-80^{\circ} \mathrm{C}$.

\subsection{DNA pulldown and on-bead sample preparation}

Oligo baits were ordered via custom synthesis from Biomers with 5'-biotinylation of the forward strand (Supporting Information Table 1). Oligos were combined with $1.5 \mathrm{X}$ molar excess of the reverse strand in $2 \mathrm{X}$ annealing buffer (20 mM TRIS, pH 8.0, $100 \mathrm{mM} \mathrm{NaCl}$ and $2 \mathrm{mM}$ EDTA) and denatured at $98^{\circ} \mathrm{C}$ for $10 \mathrm{~min}$. Oligos were allowed to anneal by cooling to room temperature overnight and subsequently stored at $-20^{\circ} \mathrm{C}$. For each pulldown reaction, $20 \mu \mathrm{L}$ bead slurry (10 $\mu \mathrm{L}$ beads) of Streptavidin-Sepharose beads (GE Healthcare) were used. According to experimental design (Fig. 1A), each pulldown was performed in duplicate for outlier calling. Label swapping was performed between replicates to eliminate labeling bias. All pulldowns from each labeling 
A

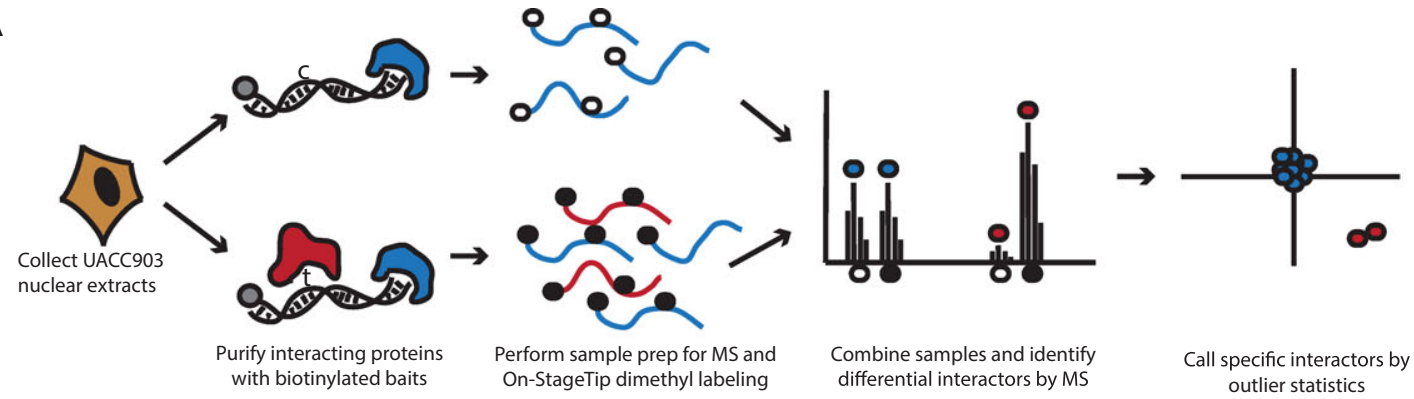

B

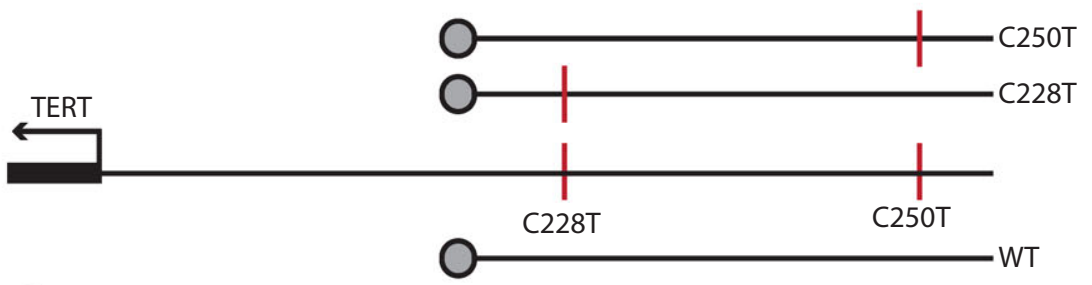

C

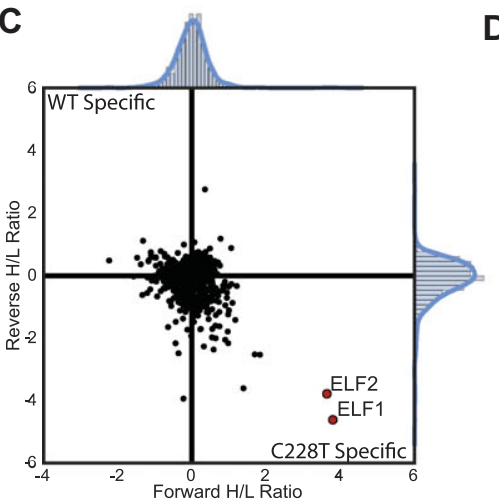

F

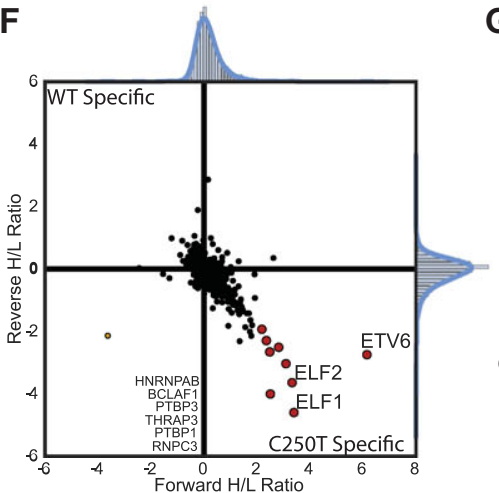

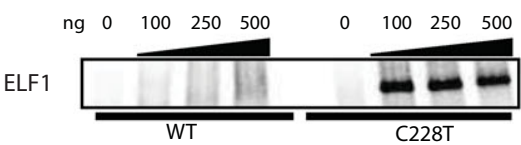

$\begin{array}{lllllllllll}\text { ng } & 0 & 50 & 100 & 250 & 500 & 0 & 50 & 100 & 250 & 500\end{array}$

GABP

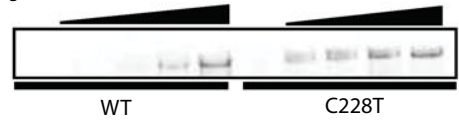

G

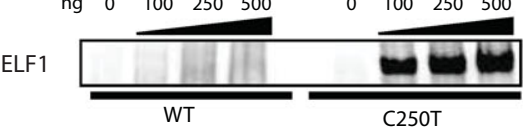

ng $\quad \begin{array}{llllllllll}0 & 50 & 100 & 250 & 500 & 0 & 50 & 100 & 250 & 500\end{array}$

GAB

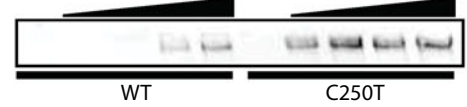

E

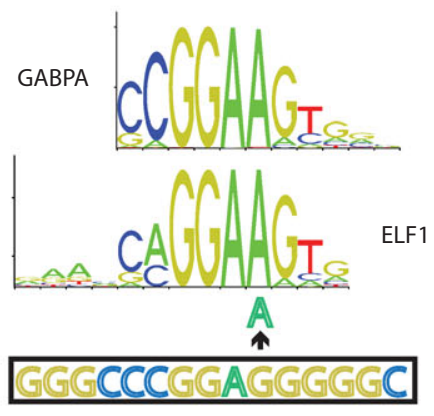

WT Sequence at C228T

H

GABPA
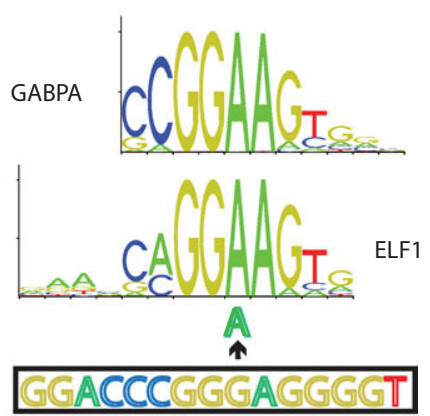

WT Sequence at C250T

Figure 1. AP-MS/MS identifies TERT promoter mutation specific interactors. (A) A workflow for MS-based identification of sequence-specific DNA interacting proteins. (B) Custom oligos were $5^{\prime}$ biotinylated and designed to cover both the C228T and C250T mutation sites. Oligos are referred to by mutation as shown in the diagram. (C) AP-MS/MS analysis of the C228T TERT promoter mutation interactors. Interactors with a ratio of at least 3 IQR (inter-quartile range) in both experiments are colored in red. Ratios are shown after log2 transformation. Labels were swapped between replicates to avoid labeling bias, hence specific interactors showing a high ratio in one experiment, and a low ratio in the other. Outlier proteins not observed consistently across experiments are noted at the bottom of the chart. (D) Band-shift experiments with the C228T TERT promoter mutation oligo. Recombinant human protein was incubated with annealed oligo and resolved on a TBE polyacrylamide gel. Band-shift experiments for each protein-oligo combination were resolved on the same gel at the same exposure. (E) JASPAR motif prediction agrees with mutation specific ELF1 and GABPA binding at C228T [29, 30]. The underlying wild-type sequence surrounding the $\mathrm{C} 228 \mathrm{~T}$ mutation is shown beneath the JASPAR motifs, and the mutation is indicated by arrow. (F) AP-MS/MS analysis of C250T TERT promoter mutation interactors. (G) Band-shift experiments with the C250T TERT promoter mutation oligo and recombinant human protein. (H) JASPAR motif prediction agrees with mutation specific ELF1 and GABPA binding at C250T. The underlying wild-type sequence surrounding the C250T mutation is indicated below the JASPAR motifs as in (E). 
reaction (forward and reverse) were performed simultaneously. Beads were washed once with $0.1 \%$ NP40 in 1X PBS and once with DNA binding buffer (DBB: $1 \mathrm{M} \mathrm{NaCl}, 0.05 \%$ NP40, 10 mM TRIS, pH 8.0 and 1 mM ETDA). Annealed oligo (500 pmol) was diluted in $600 \mu \mathrm{L}$ DBB final volume and rotated for $30 \mathrm{~min}$ at $4^{\circ} \mathrm{C}$. Subsequent steps were all carried out at $4^{\circ} \mathrm{C}$. Beads with immobilized oligonucleotides were washed once with DBB and twice with protein binding buffer (PBB: $150 \mathrm{mM} \mathrm{NaCl}, 0.25 \%$ NP40, $50 \mathrm{mM}$ TRIS, pH 8.0, EDTA-free complete protease inhibitors, and $1 \mathrm{mM}$ DTT). Nuclear extracts $(500 \mu \mathrm{g})$ and $10 \mu \mathrm{g}$ of competitor DNA ( $5 \mu \mathrm{g}$ poly-dIdC, $5 \mu \mathrm{g}$ poly-dAdT) were added to beads in a $600 \mu \mathrm{L}$ final volume. For all AP-MS/MS analyses, nuclear extract from UACC 903 cells (C228T-positive) was used. Beads were incubated for $90 \mathrm{~min}$ on a rotation wheel at $4^{\circ} \mathrm{C}$. The beads were then washed three times with PBB and two times with 1X PBS. All supernatant was carefully removed with a syringe. The proteins were reduced in elution buffer $(2 \mathrm{M}$ urea, $10 \mathrm{mM}$ DTT, and $100 \mathrm{mM}$ ammonium bicarbonate) for $20 \mathrm{~min}$ with shaking at room temperature. Samples were alkylated by addition of $50 \mathrm{mM}$ iodoacetamide (IAA) in the dark with shaking at room temperature for $10 \mathrm{~min}$. Proteins were then subjected to on-bead trypsin digestion $(0.25 \mu \mathrm{g})$ for $2 \mathrm{~h}$ at room temperature plus shaking. The supernatant was transferred to a new tube and digested with an additional $0.1 \mu \mathrm{g}$ trypsin overnight.

\subsection{On-stagetip dimethyl labeling}

Tryptic peptides were purified on C18 stage-tips (without acidification) as described previously [24]. Buffer A was $0.1 \%$ formic acid and Buffer B was $80 \%$ acetonitrile and $0.1 \%$ formic acid. On-StageTip dimethyl labeling was performed as described previously [25]. Briefly, $300 \mu \mathrm{L}$ of labeling reagent (16.2 $\mu \mathrm{L} 37 \% \mathrm{CH} 2 \mathrm{O}$ (light) or $30.0 \mu \mathrm{L} 20 \% \mathrm{CD} 2 \mathrm{O}$ (medium) plus $6 \mathrm{mg}$ sodium cyanoborohydride in $3 \mathrm{~mL}$ of labeling buffer [10 mM NaH2PO4, $35 \mathrm{mM} \mathrm{Na2HPO4])} \mathrm{was} \mathrm{applied}$ to the StageTip and spun through at $2200 \times \mathrm{g}$ for $10 \mathrm{~min}$. StageTips were then washed once with $100 \mu \mathrm{L}$ of Buffer A and stored at $4^{\circ} \mathrm{C}$ for MS analysis.

\subsection{Mass spectrometry analysis}

Labeled samples were eluted from the StageTips with $30 \mu \mathrm{L}$ of Buffer B while combining the respective light and medium labeled pairs into the same tube. Acetonitrile was evaporated by SpeedVac centrifuge at room temperature. After resuspension with $7 \mu \mathrm{L}$ of Buffer A, $5 \mu \mathrm{L}$ of sample was loaded onto a $30 \mathrm{~cm}$ column (heated at $40^{\circ} \mathrm{C}$ ) packed in-house with $1.8 \mu \mathrm{m}$ Reprosil-Pur C18-AQ (Dr Maisch). The peptides were eluted from the column using a gradient from 7 to $32 \%$ Buffer B in Buffer A over $120 \mathrm{~min}$ at flow rate $250 \mathrm{~nL} / \mathrm{min}$ using an Easy-nLC 1000 (Thermo Fisher Scientific).

C228T, C250T, and 2MT samples were eluted and sprayed directly into a Thermo Fisher QExactive mass spectrome- ter. The mass spectrometer was operated in top ten datadependent acquisition mode. Target values for full MS were set to 3e6 AGC target and a maximum injection time of $20 \mathrm{~ms}$. Full MS were recorded at a resolution of 70000 over a scan range of 300-1650 m/z. Target values for MS/MS were set at 1e5 AGC target with a maximum injection time of $120 \mathrm{~ms}$. The MS/MS spectra were recorded at a resolution of 17500 . The isolation width was set to $3.0 \mathrm{~m} / z$, the collision energy to $\mathrm{NCE}=25$, and the intensity threshold to 8.3e2. Dynamic exclusion was enabled for $20 \mathrm{~s}$. Peptides with single or unknown charge state were excluded for MS/MS analysis.

C228T+ETS samples were eluted and sprayed directly into a Thermo Fisher Orbitrap Fusion Tribrid mass spectrometer. Target values for full MS were set to 4e5 AGC target and a maximum injection time of $50 \mathrm{~ms}$. Full MS were recorded at a resolution of 120000 at a scan range of $400-1500 \mathrm{~m} / \mathrm{z}$. Most intense precursors with a charge state between 2 and 7 were selected for MS/MS analysis, with an intensity threshold of 5000 and dynamic exclusion for $60 \mathrm{~s}$. Target values for MS/MS were set at 1e4 AGC target with a maximum injection time of $35 \mathrm{~ms}$. Ion trap scan rate was set to 'rapid', with an isolation width of $1.6 \mathrm{~m} / z$ and collision energy of $35 \%$.

\subsection{Data analysis}

Raw MS spectra were analyzed using MaxQuant software (version 1.5.1.0) with standard settings [26,27]. For dimethyl labelled samples, the respective built in N-terminal and lysine modification for dimethyl labeling was specified under "labels". Carbamidomethylation was specified as a fixed modification on cysteines. N-terminal acetylation and methionine oxidation were allowed as variable modifications. Trypsin was selected as specific enzyme, and two missed cleavages were allowed. Data was searched against the human UniProt database (fasta file downloaded 2014.09.03) using the integrated search engine. The search was performed with a mass tolerance of $4.5 \mathrm{ppm}$ mass accuracy for the precursor ion and $20 \mathrm{ppm}$ for fragment ions. Peptides and proteins were both accepted at an FDR of 0.01. For quantification, at least two ratio counts were required. Protein identifications and calculated ratios are included as the proteinGroups output file from MaxQuant analysis (Supporting Information Table 2). Plots were generated with Python essentially as described previously [28]. Briefly, protein identifications were filtered for contaminants and reverse hits. Proteins groups were required to have two identified peptides, of which at least one was unique, to be considered as identified. The required outlier significance was 3.0 IQRs (inter-quartile range) for both forward and reverse experiments.

\subsection{Band-shift experiments}

Band-shift experiments were performed by incubating $20 \mathrm{fmol}$ of biotin labeled double stranded oligos with recombinant human ELF1 protein (Origene, TP760629) or 
recombinant GABPA/B (Abnova, GABPA: H00002551-P01, GABPB: H00002553-P01) in a total volume of $20 \mu \mathrm{L}$ of protein binding buffer (PBB: $150 \mathrm{mM} \mathrm{NaCl}, 0.25 \%$ NP40, 50 mM TRIS, pH 8.0, and $1 \mathrm{mM}$ DTT) for $30 \mathrm{~min}$. For GABP experiments, GABPA and GABPB were mixed at equimolar concentrations for $20 \mathrm{~min}$ at room temperature prior to addition of the oligo, in GABP experiments, the molecular weights listed in the figures refers to the total molecular weight of protein used (GABPA/B combined). The resulting protein complexes were resolved on 4-20\% TBE gels (Biorad) in a Mini-PROTEAN tetra cell (Biorad) at $100 \mathrm{~V}$ for approximately $3 \mathrm{~h}$ in $1 \mathrm{X}$ TBE. Samples were transferred onto a nylon membrane (Biodyne) in a Trans-Blot Turbo Transfer semi-dry transfer system (Biorad) at $400 \mathrm{~mA}$ for $10 \mathrm{~min}$. Membranes were UV cross-linked and oligos were detected using streptavidin-HRP conjugate and a chemiluminescent substrate (Chemiluminescent Nucleic Acid Detection Module, Pierce). Raw data from all bandshift experiments reported in this study is presented in Supporting Information Fig. 1.

\subsection{Western blotting}

DNA pulldowns were performed as described above. After the final PBS wash, samples were resuspended in MilliQ water plus $1 \mathrm{X}$ sample buffer and boiled at $95^{\circ} \mathrm{C}$ for $10 \mathrm{~min}$. Samples were then resolved on a poly-acrylamide gel, transferred to a nitrocellulose membrane, blocked with $5 \%$ milk, and incubated with primary antibody at $4^{\circ} \mathrm{C}$ overnight (ELF1: Santa Cruz, sc-631; GABPA: Santa Cruz, sc-22810). Samples were then incubated with HRP-conjugated secondary antibody (Dako) for $1 \mathrm{~h}$ at room temperature, and imaged using an ECL Western Blotting Substrate (Pierce).

\subsection{RNA and genomic DNA extraction}

RNA was extracted using an RNeasy Plus Mini Kit (Qiagen). Genomic DNA was isolated using the ZR genomic DNA ${ }^{\mathrm{TM}}$ kit (D3050, ZYMO Research) and assessed by Nanodrop 8000 (Thermo Scientific).

\subsection{Long PCR and sequencing of TA clones}

PCR was carried out using genomic DNAs from 7 UACC melanoma cell lines as indicated in Fig. 4A. The PCR primers used were: GGGCCGCAGCTGCTCCTTGTCG and CAGGCCGGGCTCCCAGTGG. PCR conditions were $98^{\circ} \mathrm{C}$ for $10 \mathrm{~min}$ to initially denature, followed by 38 cycles of: $98^{\circ} \mathrm{C}$ for $30 \mathrm{~s}$, $60^{\circ} \mathrm{C}$ for $30 \mathrm{~s}$, and $72^{\circ} \mathrm{C}$ for $90 \mathrm{~s}$, with a $7-\mathrm{min}$ final extension at the end. The PCR products were cloned into TA vector (Life Technologies). Single colonies of bacteria were selected and PCR sequenced in 96-well plate format. The PCR reaction was performed in the same manner as above. Sanger sequencing was used to determine the sequences of the PCR product.

\subsection{Real-time quantitative PCR and allelic specific TERT expression}

Gene expression levels were quantified by quantitative realtime PCR using TaqMan assays for TERT (Hs00972656_m1), ELF1 (Hs01111177_m1), ELF2 (Hs00959420_g1), GABPA (Hs01022016_m1), and GAPDH (cat\#4333764) from Life Technologies. siRNA knockdown experiments were performed using siRNAs purchased from Dharmacon targeting GABPA (D-001810-01-05), ELF1 (L-012669-00-0005), and ELF2 (L-012754-00-0005). A non-targeting scrambled siRNA was used as the control (D-001810-01-05). Gene expression levels of TERT, ELF1 and ELF2 were normalized to GAPDH. Allele specific TERT expression was determined using an allelic discrimination TaqMan assay for rs2736098 (assay C_26414916_20, Life Technologies), and the gene expression of each allele of TERT was also normalized to the gene expression of GAPDH. Each experiment was performed in triplicate and repeated three times.

\section{Results and discussion}

\subsection{ELF1 binds specifically to both TERT promoter mutations}

We used an AP-MS/MS based workflow to identify the proteome-wide interactomes of both TERT promoter mutations (Fig. 1A). Oligo baits were designed to encompass both mutation sites concurrently, with one mutation per oligo (Supporting Information Table 1, Fig. 1B). For AP-MS/MS analysis, we used UACC903 metastatic melanoma derived cell lines, characterized as C228T-positive (unpublished data). We identified ELF1 and ELF2 as specific interactors at both the C228T and C250T mutation sites and ETV6 as a specific interactor at the C250T mutation site (Fig. 1C and F). We confirmed the specificity and robustness of the ELF1 interaction via bandshift with recombinant protein (Fig. 1D and G). JASPAR in silico motif prediction agrees with mutation specific binding for ELF1 and GABPA (Fig. 1E and H) $[29,30]$. However, we did not observe a specific GABP interaction with either TERT promoter mutation by MS analysis. Intriguingly, band-shift experiments using pure, recombinant GABP revealed a subtle preference for the $\mathrm{C} 228 \mathrm{~T}$ and C250T mutations over the wild-type sequence (Fig. 1D and G). This potentially indicates that the stabilizing effect of a single mutation (and thus a single canonical motif) is sufficient to relatively increase recombinant GABP binding in vitro. Indeed, comparison between previous GABP electrophoresis analysis and TERT promoter bandshift experiments suggests that the low-mobility complex we observed is indeed the GABPA/B heterotetramer [31]. However, GABP concentrations in nuclear lysates are likely far lower than those used in bandshift experiments, which explains why GABP was not identified by AP-MS/MS; our data thus suggests that ELF1 is a significant, specific interactor of single ETS motifs 
A

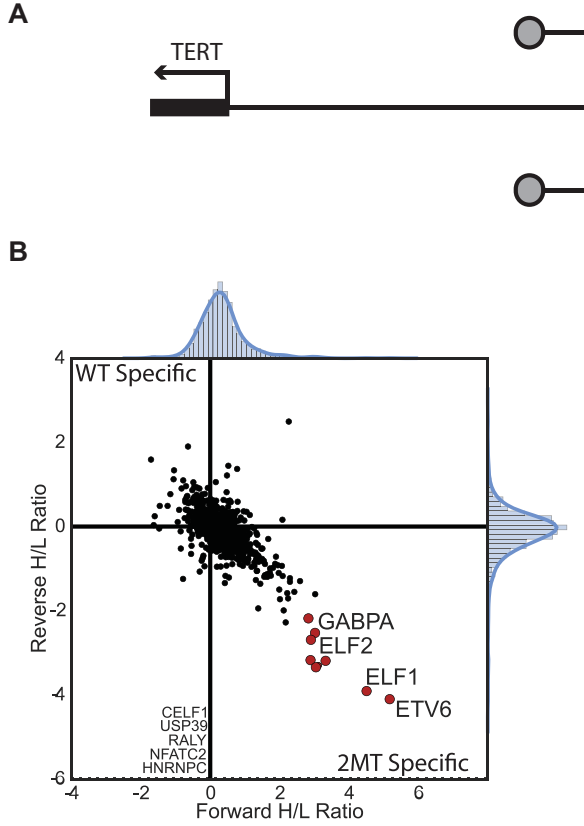

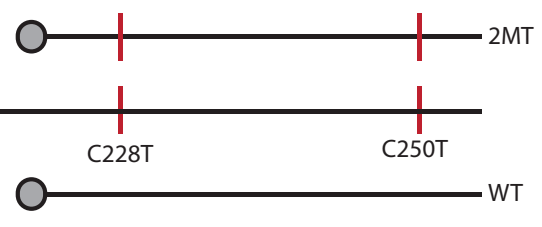

C
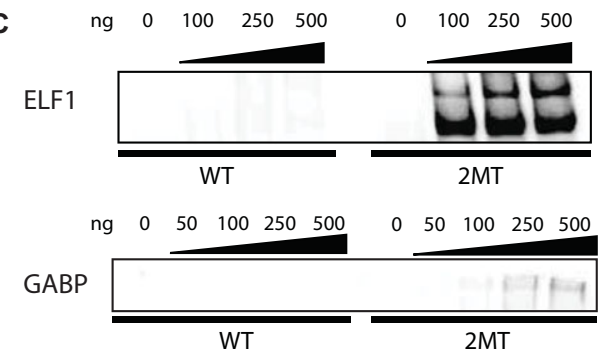

D

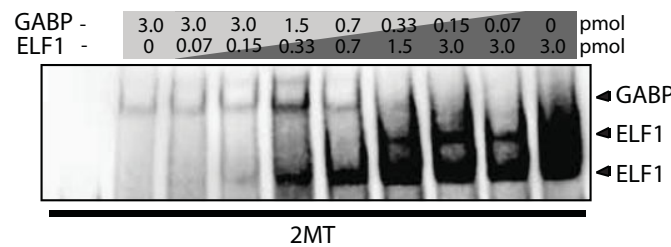

Figure 2. Analysis of protein binding patterns at the TERT promoter double mutation. (A) Oligos were designed as before, but encompassing both common TERT promoter mutations. (B) AP-MS/MS analysis of the double mutation (2MT) oligo containing both TERT promoter mutations. (C) Band-shift experiments with the 2MT TERT promoter oligo and recombinant human protein. (D) Competitive binding experiments between ELF1 and GABP at the 2MT TERT promoter mutation oligo. Molarity for GABP indicates the calculated molarity of the tetrameric unit. created by recurrent mutations in the TERT promoter. However, TERT promoter mutation-specific GABP binding at single novel ETS motifs, while present, appears to be of lower affinity.

\subsection{Analysis of transcription factor binding at the TERT promoter double mutation}

We were interested in the observed mutual exclusivity of the TERT promoter C228T and C250T mutations; are they merely functionally redundant, or are there combinatorial binding modes generated by the second ETS motif that preclude its appearance in vivo? To address this, we applied our MS workflow to the TERT promoter double mutation sequence (Fig. 2A). We observed the same set of ETS-family transcription factors as significant interactors: ELF1, ELF2, and ETV6 (Fig. 2B). We were again able to confirm specific ELF1 binding by band-shift assay (Fig. 2C). Moreover, with the double mutation sequence, we observed GABPA as a mutation-specific interactor by MS analysis and by band-shift assay (Fig. 2B and C). Importantly, this agrees with a model where heterotetrameric binding at two precisely spaced ETS motifs stabilizes GABP binding relative to the wild-type sequence. Furthermore, our data suggests that the two novel ETS motifs on the 2MT oligo may facilitate a greater degree of GABP stabilization or occupancy compared with either C228T or C250T independently.

We conducted competitive binding assays by band-shift and observed substantially greater ELF1 binding compared with GABP in equimolar conditions (assuming tetrameric binding) (Fig. 2D). Of note, we observed doublet bands for
ELF1 corresponding to monomeric binding or simultaneous binding by two ELF1 molecules, but this doublet pattern was not seen for GABP. Our data therefore supports the robust monomeric binding of ELF1 and stable but lower affinity heterotetrameric binding of GABP to the 2MT oligo. We did not observe any other differential binding events at the double mutation, indicating that both mutations are likely functionally redundant. Therefore, the double mutation is likely not strongly selected for during cancer progression compared with either mutation independently.

\subsection{GABP binding at novel and native ETS motifs excludes ELF1 and activates TERT expression}

To resolve the discrepancy between ELF1 binding at TERT promoter mutations in vitro and its lack of transcriptional effect in vivo, we again performed AP-MS/MS analysis of TERT promoter mutations, utilizing the C228T mutation with two upstream native ETS motifs as a representative case (Fig. 3A)[15]. This oligo design facilitated study of combinatorial binding with novel and native ETS motifs. Indeed, recent work indicates that in the case of 250T, GABP still binds using ETS-195 and ETS-200 instead of upstream ETS motifs [15]. These native motifs (ETS-195, ETS-200) represent a different spatial architecture with either C228T or C250T compared to C228T and C250T with each other (in the case of the 2MT oligo), which likely has implications for the mutual exclusivity of C228T and C250T mutations in vivo. For $\mathrm{C} 228 \mathrm{~T}+\mathrm{ETS}$, we observed robust and specific binding of GABP by MS analysis, and we could confirm the relative specificity of this binding by band-shift assay (Fig. 3B and C). 
A

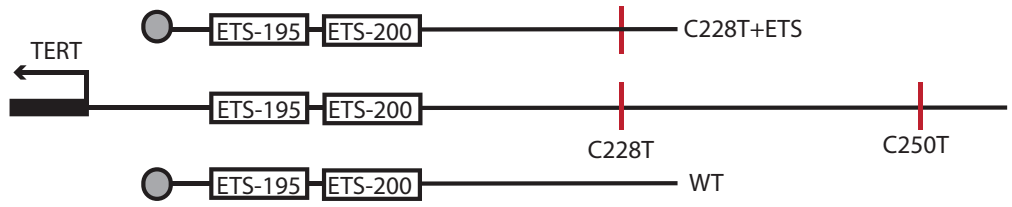

B

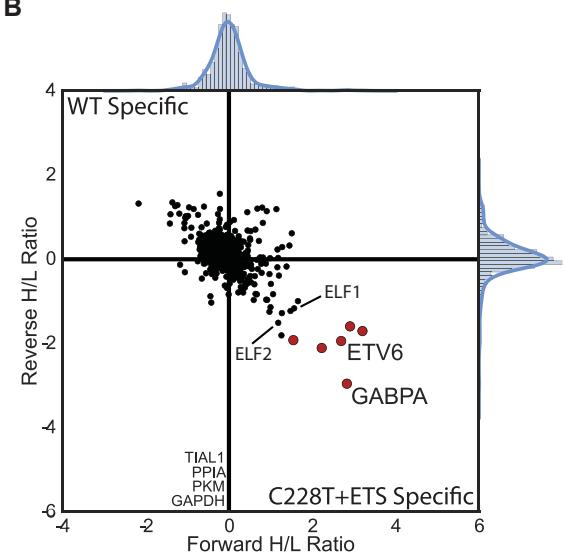

D

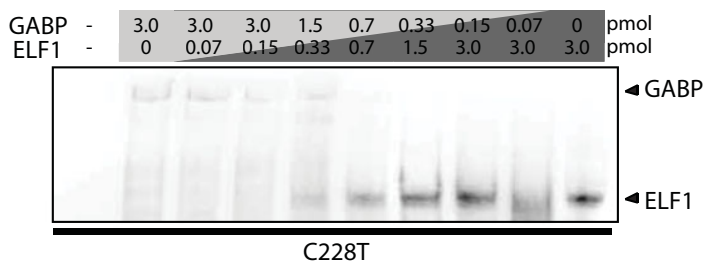

E

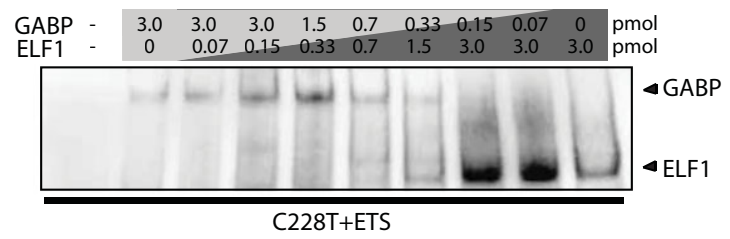

We observed increased GABP binding concurrent with a reduction in specific ELF1 and ELF2 binding; indeed, neither reached significance at thresholds chosen in our previous analysis. By competition band-shift assay, we were able to directly compare ELF1 and GABP binding using both the $\mathrm{C} 228 \mathrm{~T}$ and the C228T+ETS oligos. With the C228T oligo, we observed relatively higher ELF1 binding compared with GABP in equimolar conditions (Fig. 3D). However, with the C228T+ETS oligo, we observed increased binding for GABP in equimolar conditions (Fig. 3E). This supports our MS analysis indicating that heterotetrameric GABP binding excludes ELF1 by occupying both the native and the novel ETS the stabilizing effect of a single TERT promoter mutation, in conjunction with binding at native ETS motifs present in $\mathrm{C} 228 \mathrm{~T}+\mathrm{ETS}$ recruits GABP binding more robustly than does 2MT, C228T or C250T. Furthermore, by western blot we were able to support our MS and band-shift data, showing motifs present in this sequence. This further indicates that
Figure 3. Heterotetrameric GABP excludes ELF1 from TERT promoter mutation binding. (A) Oligos were designed as before, but encompassing the C228T mutation plus two additional upstream ETS motifs. (B) AP-MS/MS analysis of the $\mathrm{C} 228 \mathrm{~T}+\mathrm{ETS}$ interactors. ELF1 and ELF2 were not significant at a 3 IQR cutoff, but were noted specifically for comparison to GABPA and previous binding ratios. (C) Band-shift experiments with the C228T+ETS TERT promoter mutation oligo. The grey line in the ELF1 bandshift indicates re-positioning of lanes; all lanes were run on the same gel, and exposures were kept uniform. (D) Competitive binding experiments between recombinant human ELF1 and GABP at the C228T TERT promoter mutation oligo. (E) Competitive binding experiments between recombinant human ELF1 and GABP at the $\mathrm{C} 228 \mathrm{~T}+\mathrm{ETS}$ TERT promoter mutation oligo. (F) Western blot analysis of GABPA and ELF1 for each oligo used in this study. All lanes are taken from the same gel at the same exposure. increased ELF1 binding over wild-type at the C228T, C250T, $2 \mathrm{MT}$, and to a lesser extent the C228T+ETS sequences, and increased GABP binding over wild-type at the C250T, 2MT, and $\mathrm{C} 228 \mathrm{~T}+\mathrm{ETS}$ sequences but not the C228T sequence (Fig. 3F). This strongly indicates that the GABP binding observed at C228T+ETS critically depends on the native ETS motif, as no specific binding is seen at C228T. Also, preferential binding at $\mathrm{C} 250 \mathrm{~T}$ but not $\mathrm{C} 228 \mathrm{~T}$, suggests GABP binding at $228 \mathrm{~T}$ versus $\mathrm{C} 250 \mathrm{~T}$ might operate under different kinetics $[15,32]$.

To study the relationship between GABP, ELF1, and ELF2 binding and TERT expression in vivo, we used a PCR-based Sanger sequencing approach to identify seven melanoma cell lines with both a heterozygous germline SNP variant (rs2736098) and a haplotype-phased heterozygous TERT promoter mutation. In these cell lines, we observed mono-allelic TERT expression in which the expressed SNP allele always correlated with a phased TERT promoter mutation (Fig. 4A). 
A

\begin{tabular}{|c|c|c|c|c|}
\hline Cell line & Expression T-allele/C-allele & Expressed allele & $\begin{array}{c}\text { TERT Promoter Mutation Strand: } \\
\text { T-allele }\end{array}$ & $\begin{array}{c}\text { TERT Promoter Mutation Strand: } \\
\text { C-allele }\end{array}$ \\
\hline UACC91 & $>100$ & T & + & - \\
\hline UACC383 & $>100$ & T & + & - \\
\hline UACC457 & $<0.01$ & C & - & + \\
\hline UACC502 & $<0.01$ & C & - & + \\
\hline UACC1592 & $>100$ & T & + & - \\
\hline UACC1940 & $>100$ & T & + & - \\
\hline UACC2331 & $<0.01$ & C & - & + \\
\hline
\end{tabular}

B

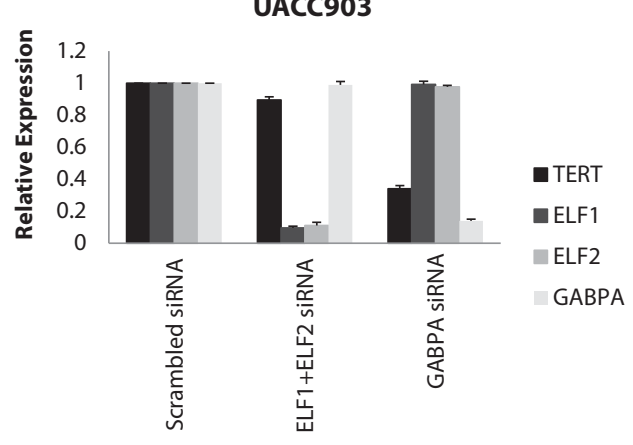

C

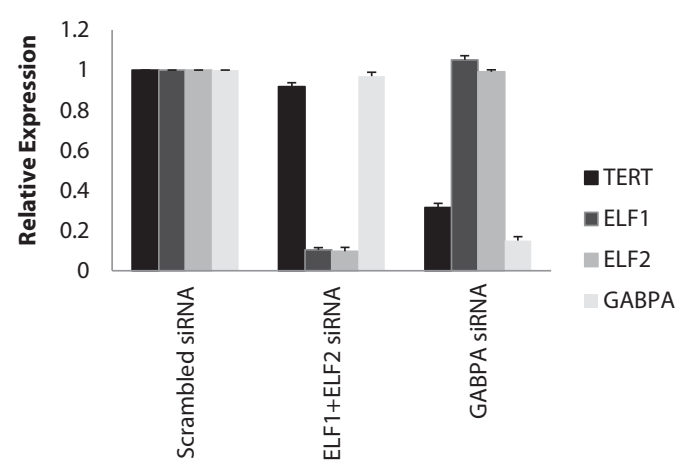

Figure 4. GABP activates mono-allelic TERT expression via promoter mutations. (A) TERT expression is mono-allelic and correlates with promoter mutation status. Mono-allelic TERT expression was assayed at rs2736098. Expression of only one allele was observed. The expressed allele correlated with a phased TERT promoter mutation as observed by PCR-based Sanger sequencing. (B) In the UACC903 melanoma cell line, GABPA knockdown substantially reduces TERT expression, while combined ELF1/2 knockdown minimally reduces TERT expression. Experiments were performed in triplicate and repeated three times. Error bars represent standard error of the mean. (C) In the UACC1113 melanoma cell line, GABPA knockdown substantially reduces TERT expression, while combined ELF1/2 knockdown minimally reduces TERT expression.

Then, we used siRNA-mediated knockdown to discern the direct transcriptional effects of ELF1/2 and GABPA on TERT expression. In two separate cell lines, we observed a substantial reduction in TERT expression upon GABPA knockdown but only minimally reduced TERT expression upon combined ELF1/2 knockdown (Fig. 4B and C). Thus, this data strongly indicates that TERT reactivation proceeds via mono-allelic TERT expression exclusive to the promoter mutation phased chromosome. Upon displacement of minimally activating ELF1 or ELF2, GABP binding induces a robust transcriptional response driving TERT expression. This study suggests a model where binding of heterotetrameric, transcriptionally active GABP at novel and native ETS motifs effectively precludes ELF1 occupancy at the TERT promoter mutations (Fig. 5).

\section{Concluding remarks}

Understanding dynamic, specific interactions between transcription factors and the cognate DNA motifs they bind is crucial towards elucidating their mechanisms of transcriptional regulation. For TERT promoter mutations, understanding these dynamic binding specificities is of particular importance as transcriptional activation is directly correlated with oncogenic outcomes. This study utilizes an unbiased proteome-wide approach to identify dynamic interplay between GABP and ELF1 via their specific interactions with the TERT promoter mutations. This study also highlights the importance of considering both native motifs and the spatial architecture of DNA baits when performing DNA-protein APMS/MS experiments. The combinatorial nature of transcription factor binding is often difficult to predict, and this study offers a cautionary note that oligo baits of different lengths or different sequence/motif compositions might produce discrepant results even for the same locus. Indeed, as this study indicates, when using oligos designed to minimally cover a SNP or a cancer mutation, biologically important interactions may be overlooked.

Although most ETS-transcription factors are reported to bind monomerically, cooperative, antagonistic, and combinatorial effects are common [33]. For example, Bell et al. use an elegant bioinformatics analysis to indicate that strong GABPA binding sites from ENCODE ChIP-seq tracks correlates with multiple motifs spaced in a manner suggesting dimerization [15,34]. This periodic feature is unique to GABPA and was not seen in ELF1 ChIP-seq tracks, suggesting that ELF1 in contrast binds monomerically. Indeed, GABP is known to be the only obligate multimer among ETS-family transcription factors $[17,19,20]$. Intriguingly, competitive binding 
A

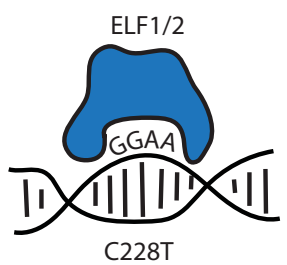

GABPA/B
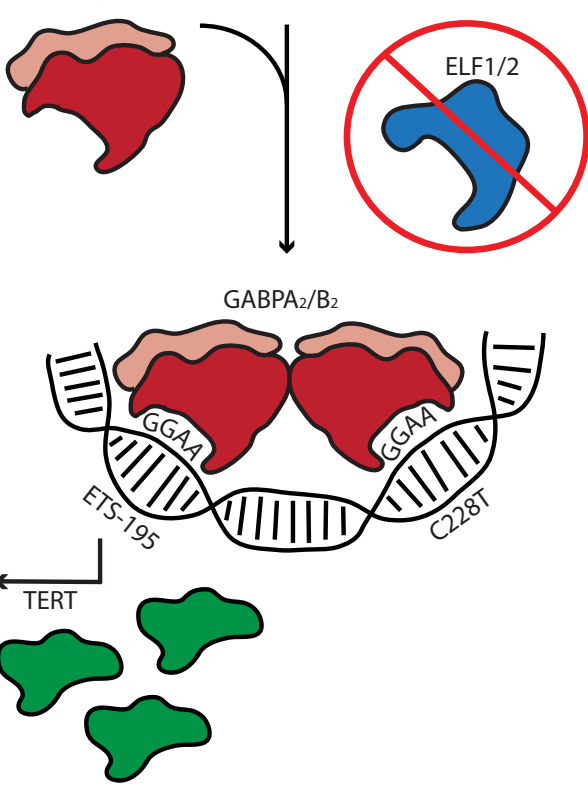

Figure 5. A model for ELF1 exclusion by heterotetrameric GABP at TERT promoter mutations. (A) ELF1 binds in vitro at novel ETS motifs created by TERT promoter mutations. However, GABP binds with high affinity at novel and native ETS motifs and excludes ELF1 from binding.

between GABP and another ETS factor, PU.1, has been seen at the CD18 promoter, yet this competition cooperatively drives CD18 expression [31]. Recent reports have indicated that diverse combinatorial effects are crucial in the activating function of the TERT promoter mutations, specifically in cooperation with native ETS-motif binding factors $[15,32]$. Although our data indicates that ELF1 knockdown has only a mild effect on downstream TERT expression (which seems to be driven predominantly by GABP), we cannot exclude the possibility of combinatorial effects with ELF1 or other natively binding factors in the TERT core promoter.

This study highlights the dynamic nature of transcription factor binding at the recurrent TERT promoter mutations and points towards a complex picture of oncogenic transcriptional regulation at this locus. We present a model where stable, heterotetrameric, transcriptionally active GABP excludes monomeric ELF1 (Fig. 5). In doing so, we confirm the identification of GABP as a mutation-specific interactor in melanoma and contribute to our knowledge of regulatory mechanisms at this important locus.
M.M. and M.V. received funding from the DevCom FP7 Marie Curie ITN. K.M.B., J.F., J.C., and T.Z. are supported by the Intramural Research Program of the Division of Cancer Epidemiology and Genetics; National Cancer Institute; National Institutes of Health.

The authors declare no conflicts of interest.

\section{$5 \quad$ References}

[1] Hanahan, D., Weinberg, R. A., Hallmarks of cancer: the next generation. Cell 2011, 144, 646-674.

[2] Low, K. C., Tergaonkar, V., Telomerase: central regulator of all of the hallmarks of cancer. Trends Biochem. Sci. 2013, 38, 426-434

[3] Wright, W. E., Piatyszek, M. A., Rainey, W. E., Byrd, W., Shay, J. W., Telomerase activity in human germline and embryonic tissues and cells. Dev. Genet. 1996, 18, 173-179.

[4] Greenberg, R. A., O'Hagan, R. C., Deng, H., Xiao, Q. et al., Telomerase reverse transcriptase gene is a direct target of c-Myc but is not functionally equivalent in cellular transformation. Oncogene 1999, 18, 1219-1226.

[5] Yin, L., Hubbard, A. K., Giardina, C., NF-кB regulates transcription of the mouse telomerase catalytic subunit. J. Biol. Chem. 2000, 275, 36671-36675.

[6] Zhang, Y., Toh, L., Lau, P., Wang, X., Human telomerase reverse transcriptase (hTERT) is a novel target of the $\mathrm{Wnt} / \beta$ catenin pathway in human cancer. J. Biol. Chem. 2012, 287, 32494-32511.

[7] Horn, S., Figl, A., Rachakonda, P. S., Fischer, C. et al., TERT promoter mutations in familial and sporadic melanoma. Science 2013, 339, 959-961.

[8] Huang, F. W., Hodis, E., Xu, M. J., Kryukov, G. V. et al., Highly recurrent TERT promoter mutations in human melanoma. Science 2013, 339, 957-959.

[9] Killela, P. J., Reitman, Z. J., Jiao, Y., Bettegowda, C. et al., TERT promoter mutations occur frequently in gliomas and a subset of tumors derived from cells with low rates of self-renewal. Proc. Natl. Acad. Sci. U S A 2013, 110, 60216026.

[10] Vinagre, J., Almeida, A., Pópulo, H., Batista, R. et al., Frequency of TERT promoter mutations in human cancers. Nat. Commun. 2013, 4.

[11] Fredriksson, N. J., Ny, L., Nilsson, J. A., Larsson, E., Systematic analysis of noncoding somatic mutations and gene expression alterations across 14 tumor types. Nat. Genet. $2014,46,1258-1263$.

[12] Weinhold, N., Jacobsen, A., Schultz, N., Sander, C. et al., Genome-wide analysis of noncoding regulatory mutations in cancer. Nat. Genet. 2014, 46, 1160-1165.

[13] Chiba, K., Johnson, J. Z., Vogan, J. M., Wagner, T. et al., Cancer-associated TERT promoter mutations abrogate telomerase silencing. eLife 2015, 4, e07918.

[14] Naxerova, K., Elledge, S. J., Taking the brakes off telomerase. eLife 2015, 4, e09519. 
[15] Bell, R. J., Rube, H. T., Kreig, A., Mancini, A. et al., The transcription factor GABP selectively binds and activates the mutant TERT promoter in cancer. Science 2015, 348, 1036-1039.

[16] LaMarco, K., Thompson, C. C., Byers, B. P., Walton, E. M. et al., Identification of Ets-and notch-related subunits in GA binding protein. Science 1991, 253, 789-792.

[17] Oikawa, T., Yamada, T., Molecular biology of the Ets family of transcription factors. Gene 2003, 303, 11-34.

[18] Sawada, J.-I., Goto, M., Sawa, C., Watanabe, H. et al., Transcriptional activation through the tetrameric complex formation of E4TF1 subunits. EMBO J. 1994, 13, 1396.

[19] Thompson, C. C., Brown, T. A., McKnight, S. L., Convergence of Ets-and notch-related structural motifs in a heteromeric DNA binding complex. Science 1991, 253, 762768.

[20] Chinenov, Y., Henzl, M., Martin, M. E., The alpha and beta subunits of the GA-binding protein form a stable heterodimer in solution. Revised model of heterotetrameric complex assembly. J. Biol. Chem. 2000, 275, 77497756 .

[21] Stern, J. L., Theodorescu, D., Vogelstein, B., Papadopoulos, N., Cech, T. R., Mutation of the TERT promoter, switch to active chromatin, and monoallelic TERT expression in multiple cancers. Genes Dev. 2015, 29.21, 22192224.

[22] Butter, F., Davison, L., Viturawong, T., Scheibe, M. et al., Proteome-wide analysis of disease-associated SNPs that show allele-specific transcription factor binding. PLOS Genet. 2012, 8, e1002982.

[23] Spruijt, C. G., Baymaz, H. I., Vermeulen, M., Identifying specific protein-DNA interactions using SILAC-based quantitative proteomics, In: Bina, M., (Ed.), Methods in Molecular Biology, vol 977: Gene Regulation. New York City: Humana Press 2013, pp. 137-157.

[24] Rappsilber, J., Mann, M., Ishihama, Y., Protocol for micropurification, enrichment, pre-fractionation and storage of peptides for proteomics using StageTips. Nat. Protoc. 2007, 2, 1896-1906.
[25] Lau, H. T., Suh, H. W., Golkowski, M., Ong, S. E., Comparing SILAC- and stable isotope dimethyl-labeling approaches for quantitative proteomics. J. Proteome Res. 2014, 13, 41644174.

[26] Cox, J., Mann, M., MaxQuant enables high peptide identification rates, individualized p.p.b.-range mass accuracies and proteome-wide protein quantification. Nat. Biotechnol. 2008, 26, 1367-1372.

[27] Cox, J., Matic, I., Hilger, M., Nagaraj, N. et al., A practical guide to the MaxQuant computational platform for SILACbased quantitative proteomics. Nat. Protoc. 2009, 4, 698-705.

[28] Hubner, N. C., Bird, A. W., Cox, J., Splettstoesser, B. et al., Quantitative proteomics combined with BAC TransgeneOmics reveals in vivo protein interactions. J. Cell. Biol. 2010, 189, 739-754.

[29] Mathelier, A., Zhao, X., Zhang, A. W., Parcy, F. et al., JASPAR 2014: an extensively expanded and updated open-access database of transcription factor binding profiles. Nucleic Acids Res. 2014, 42, D142-147.

[30] Sandelin, A., Alkema, W., Engstrom, P., Wasserman, W. W., Lenhard, B., JASPAR: an open-access database for eukaryotic transcription factor binding profiles. Nucleic Acids Res. 2004, 32, D91-D94.

[31] Rosmarin, A. G., Caprio, D. G., Kirsch, D. G., Handa, H. et al., GABP and PU.1 compete for binding, yet cooperate to increase CD18 (beta 2 leukocyte integrin) transcription. J. Biol. Chem. 1995, 270, 23627-23633.

[32] Li, Y., Zhou, Q. L., Sun, W., Chandrasekharan, P. et al., Noncanonical NF-kappaB signalling and ETS1/2 cooperatively drive C250T mutant TERT promoter activation. Nat. Cell. Biol. 2015, 17, 1327-1338.

[33] Li, R., Pei, H., Watson, D. K., Regulation of Ets function by protein - protein interactions. Oncogene 2000, 19, 65146523.

[34] Consortium, E. P., Birney, E., Stamatoyannopoulos, J. A., Dutta, A. et al., Identification and analysis of functional elements in $1 \%$ of the human genome by the ENCODE pilot project. Nature 2007, 447, 799-816. 